\title{
Hacia la Clase Inversa. Una Experiencia de Aprendizaje de la Química y de Desarrollo de Competencias en el Primer Curso de Grado en Ingenierías
}

\author{
Isabel Morera Bertomeu
}

imorera@upvnet.upv.es

GRUPO DE INNOVACIÓN E INVESTIGACIÓN EN METODOLOGÍAS ACTIVAS (GIIMA)

Departamento de Química

Universitat Politècnica de València

Camino de Vera s/n. 46071-València

\begin{abstract}
The flipped classroom model is based on the proper use of technology as a vehicle for active learning and teaching-centered design to promote the work of the student. In this model, students put more effort and commitment during the learning process, outside and inside the classroom, resulting in a more consolidated and durable learning, as well as the exercise and achievement of competences. This work presents the results of an innovative experience in flipped learning, implemented by a teacher belonging to the Grupo de Innovación e Investigación en Metodologías Activas (GIIMA). It's a learning experience of Chemistry in the first degree course of Engineering in Industrial Technologies, which is taught in the Escuela Técnica Superior de Ingenieros Industriales of the Universitat Politècnica de València. This communication describes the motivations to develop this approach and the details of its design. Also, the analysis of the results and the opinions of students are discussed.
\end{abstract}

Keywords: flipped classroom, flipped learning, flip theaching, active learning, technology as an aid to learning, competences, first degree course, Chemistry in Engineering studies.

\section{Resumen}

El modelo de la clase inversa se basa en el uso apropiado de la tecnología como vehículo para el aprendizaje activo y en el diseño pedagógico centrado en promover el trabajo del alumno. En este modelo, los estudiantes ponen más esfuerzo y compromiso durante el proceso de aprendizaje, fuera y dentro del aula, lo que se traduce en un aprendizaje más consolidado y duradero, así como en el ejercicio y desarrollo de competencias. En esta comunicación se presenta el resultado de la experiencia innovadora, en formato de clase inversa, que ha implementado una profesora perteneciente al Grupo de Innovación e Investigación en Metodologías Activas (GIIMA). Se trata de una experiencia de aprendizaje de la Química en el primer curso de Grado de Ingeniería en Tecnologías Industriales, que se imparte en la Escuela Técnica Superior de Ingenieros Industriales de la Universitat Politècnica de València. Se describen los motivos por los que se decide tomar esta 
Hacia la Clase Inversa. Una Experiencia de Aprendizaje de la Química y de Desarrollo de Competencias en el Primer Curso de Grado en Ingenierías

iniciativa, los detalles del diseño de la misma, así como el análisis de los resultados y las reflexiones y opiniones de los estudiantes.

Palabras clave: clase inversa, aprendizaje activo, tecnología al servicio del aprendizaje, competencias, primer curso de Grado, Química en estudios de Ingeniería .

\section{Introducción}

La clase inversa o flipped classroom/flipped learning ${ }^{l}$ (Tourón, 2015) es un tema de gran actualidad, aunque no se trata de nada nuevo. Tiene su origen en la década de los 90 en Harward, cuando Eric Mazur desarrolla Peer Instruction (Mazur, 1996) que incorpora una técnica de enseñanza denominada "Just-in-Time" que permite al profesor obtener retroalimentación de los estudiantes el día antes de la clase, de modo que puede diseñar estrategias y actividades adaptadas de forma personalizada a las necesidades de los mismos para una mejor comprensión del contenido. Esta metodología, denominada Blended Learning por combinar obtención de información on-line y trabajo activo e inductivo en clase, fue la precursora del modelo de aprendizaje inverso.

El término flipped classroom fue acuñado por Jonathan Bergmann and Aaron Sams (Bergmann, 2012), profesores de Química en Woodland Park (Colorado), quienes con intención de ayudar a los estudiantes que con relativa frecuencia perdían algunas clases por razones ajenas a su voluntad, decidieron grabar y distribuir sus grabaciones entre sus estudiantes. Rápidamente observaron en esta acción un posible modelo con gran potencial pedagógico para satisfacer las necesidades de aprendizaje de cada estudiante, y a la vez disponer de más tiempo en el aula para atender a todos de una manera más individualizada.

El aprendizaje inverso se basa en un enfoque pedagógico que transfiere fuera del aula el trabajo de determinados procesos de aprendizaje y utiliza el tiempo de clase, apoyándose en la experiencia del docente, para facilitar y potenciar otros procesos de adquisición y práctica de conocimientos (Bergmann, 2012). La asistencia a clase es fundamental ya que en ella se realizan actividades de aprendizaje que requieren interacción y participación de los estudiantes asesorados y guiados por el profesor. No se trata de elaborar y distribuir una serie de contenidos multimedia para que los estudiantes aprendan por si solos (Bergmann, 2011), sino de un enfoque integral que combina la instrucción directa con métodos constructivistas, actuaciones de compromiso e implicación de los estudiantes con el contenido del curso y la mejora de su comprensión conceptual (Tourón, 2013). Si esta acción integral se desarrolla convenientemente se recorren todas las fases de un ciclo de aprendizaje como el que sugiere la propia taxonomía de Bloom (Anderson, 2001; Bloom, 1956).

\footnotetext{
${ }^{1}$ Los términos flipped classroom o flipped learning se suelen utilizar indistintamente, aunque el segundo abarca un conjunto de elementos más amplio que la flipped classroom. Puede consultarse en: http://www.thedailyriff.com/articles/the-flipped-class-manifest-823.php [Consultado: 1de abril de 2016]
}

(c) EY-NC-ND 2016, Universitat Politècnica de València 
El modelo de la clase inversa se basa en el uso apropiado de la tecnología como vehículo para el aprendizaje activo y en el diseño pedagógico centrado en promover el trabajo del alumno de manera prioritaria y por tanto, pone el acento de la acción de aprender sobre el único que debe recaer: el estudiante. Este enfoque pedagógico se configura como un modelo que permite disponer de suficiente tiempo para garantizar la práctica y la adquisición de diversas habilidades personales y sociales sin entrar en conflicto con la adquisición de los conocimientos que configuran los programas de las materias que se incluyen en los planes de estudio. Es más, el modelo de clase inversa produce más y mejor trabajo de los estudiantes fuera y dentro del aula y se traduce en un aprendizaje más consolidado y duradero al poner más esfuerzo y compromiso durante el proceso, que facilita el ejercicio y el desarrollo de competencias, y que marcará la diferencia entre el éxito y el fracaso en las instituciones educativas del siglo XXI (Tourón, 2015).

En esencia, la clase inversa proporciona un cambio de roles: del estudiante, más centrado en el aprendizaje de los contenidos que han sido diseñados por el profesor, y del profesor, facilitador de la participación en el aprendizaje activo de sus estudiantes en el aula y dinamizador de discusiones y desarrollo de actividades aplicadas que fomentan la exploración, la articulación y aplicación de ideas.

Lejos de la creencia de que la clase inversa anula la profesión del docente, la implementación de la misma revitaliza la función de los profesores. Los profesores son más necesarios que nunca en un modelo educativo basado en el desarrollo de competencias como el trabajo autónomo, la organización del tiempo, la resolución de problemas, el trabajo en equipo y tantas otras que son exigencias del mundo en el que nuestros estudiantes van a desenvolverse (Tourón, 2014).

Es por ello que, movida por el interés de proporcionar a mis estudiantes oportunidades para estimular la motivación, favorecer la implicación, obtener mejores resultados de aprendizaje y desarrollar diferentes competencias, me he planteado iniciar una experiencia de implementación de la clase inversa en la asignatura de Química de primer curso del Grado de Ingeniería en Tecnologías Industriales (GITI), en la Escuela Técnica Superior de Ingenieros Industriales (ETSII) de la Universitat Politècnica de València (UPV). Ello aportará, si cabe, valor añadido al uso de todas las estrategias y técnicas activas, que desde hace años se vienen implementando en esta asignatura, basadas en la evidencia del efecto positivo que el uso de las metodologías centradas en el aprendizaje produce sobre el modo de aprender y el rendimiento de los estudiantes (Gargallo, 2014; Gargallo, 2015).

En esta comunicación se presenta el resultado de la experiencia innovadora, en formato de clase inversa, que ha implementado una profesora perteneciente al Grupo de Innovación e Investigación en Metodologías Activas (GIIMA), un Equipo de Innovación y Calidad Educativa (EICE) de la UPV. 
Hacia la Clase Inversa. Una Experiencia de Aprendizaje de la Química y de Desarrollo de Competencias en el Primer Curso de Grado en Ingenierías

\section{Objetivos}

El objetivo general es investigar el efecto que tiene la transformación de la clase al formato de clase inversa sobre el aprendizaje de los estudiantes, iniciando la experiencia con una parte de la asignatura. Los objetivos específicos son:

- Aumentar el protagonismo del estudiante potenciando el trabajo autónomo.

- Diseñar el proceso que incluye la creación del material, de las actividades a realizar fuera y dentro del aula y la evaluación de las mismas.

- Provocar la reflexión sobre cómo se aprende mejor.

- Recoger la opinión de los estudiantes.

- Analizar los aspectos positivos y de mejora del proceso.

- Obtener conclusiones para, en caso de necesidad, reformular el proceso.

\section{Contexto, diseño y desarrollo de la experiencia}

\subsection{El contexto de la asignatura}

La experiencia se ha llevado a cabo, durante el curso 2015-16, en la asignatura de Química del título de GITI que se imparte la ETSII de la UPV. Como puede verse en la Figura 1 se trata de una asignatura cuatrimestral, que se imparte en el segundo cuatrimestre en primer curso, tiene asignados 6 créditos ECTS y se incluye como formación básica. El número de alumnos matriculados es de 280 distribuidos en 5 grupos de docencia de aula; la innovación se ha desarrollado en el grupo de valenciano que tiene matriculados 41 estudiantes.

\section{PRIMER CURSO}

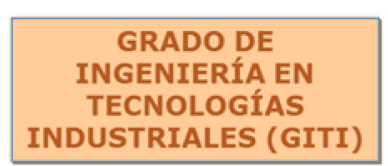

\section{FORMACIÓN} BÁSICA

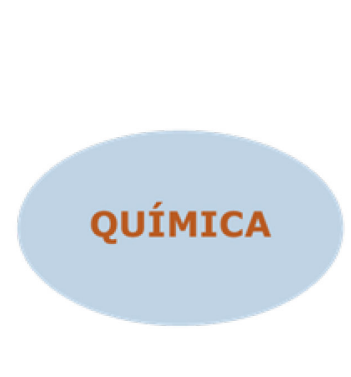

ESCUELA TÉCNICA SUPERIOR DE INGENIEROS INDUSTRIALES (ETSII)

\section{CRÉDITOS ECTS}

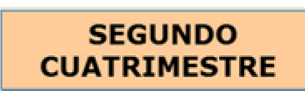

CUATRIMESTRE
5 GRUPOS DE DOCENCIA EN AULA

Fig. 1 Características de la asignatura en la que se lleva a cabo la experiencia 
En base a los datos recogidos durante varios años sobre el perfil de los estudiantes que acceden a la titulación de GITI se puede afirmar que proceden de diferentes opciones de Bachillerato y poseen niveles de formación en Química muy heterogéneos, ya que muchos de ellos no la han cursado previamente. Por ello, bastantes estudiantes se encuentran en una situación a la que les resulta difícil adaptarse, bien porque no es acorde a sus expectativas o porque les falta, en ocasiones, el nivel formativo básico en la materia. Esto les lleva, en algunos casos, a entrar en un círculo que empieza con la pérdida de interés por el aprendizaje, sigue con la desmotivación y la falta de asistencia a clase y termina con el abandono de la asignatura.

Ante esta situación, se lleva varios años implementando una serie de estrategias y técnicas activas con el fin de favorecer la integración, estimular la motivación y la implicación en el aprendizaje de los estudiantes desde los primeros días del inicio del curso. Además de disponer de una serie de cursos MOOC propedéuticos de Química que les permite adquirir la formación previa en base a sus necesidades específicas, se utilizan metodologías activas que incluyen, entre otras técnicas, clases magistrales participativas, trabajo autónomo y trabajo cooperativo en equipos. Se les proporciona, no sólo espacios de aprendizaje en el aula, sino también materiales de apoyo al aprendizaje como documentos electrónicos de las diapositivas utilizadas, libros de ejercicios resueltos y propuestos para resolver en clase y en equipos, guiones de prácticas, etc.

Cuando se analizan los datos del perfil del alumnado del grupo de valenciano, destaca que un porcentaje muy elevado ( $70 \%$ en promedio, según los cursos académicos) no ha estudiado Química en el Bachillerato. Practicamente la totalidad proceden de poblaciones con un número limitado de centros que imparten el Bachillerato y no tienen muchas posibilidades de elegir las asignaturas que desean o que consideran necesarias en función de sus preferencias por estudiar uno u otro título Universitario.

Ante esta observación, para que se impliquen desde el inicio de las clases, aprovechen su potencial y se motiven a medida que avanzan en el aprendizaje, se ha decidido, además, introducir algunas estrategias y tareas específicas como:

- Uso de "la pregunta como recurso pedagógico" en la clase magistral participativa.

- Fomento del trabajo autónomo con retroalimentación rápida: el profesor revisa y devuelve con indicaciones a los alumnos que así lo solicitan, los problemas que, de manera individual, terminen de resolver en casa después de haber planteado su resolución en clase trabajándolos en los equipos.

- Realización de tutorías programadas en el aula semanalmente (dos o tres horas), a las que voluntariamente acuden los alumnos, de forma individual o los equipos de trabajo, con el fin de proporcionarles un espacio donde poder resolver sus dudas, o trabajar en presencia del profesor las tareas que han de desarrollar en grupo.

Todo esto permite realizar un seguimiento muy personalizado del trabajo que van realizando. Esta tarea es especialmente importante en primer curso de educación superior, momento clave para reorientar los hábitos de los estudiantes con el fin de introducirlos en la 
dinámica del trabajo diario y del aprendizaje autónomo, que es el primer paso para el éxito académico.

En este contexto, y animada por la ambiciosa iniciativa del Vicerrectorado de las Tecnologías de la Información y de las Comunicaciones de transformación progresiva en la UPV de las clases al formato de clase inversa en el curso 2015-16, me planteé llevar a cabo una experiencia en una parte del contenido de la asignatura de Química, con el fin de motivar aun más al alumnado e introducirlo, desde el primer curso, en la dinámica del aprendizaje activo y el desarrollo de competencias.

\subsection{El diseño de la experiencia}

La docencia inversa se apoya en dos aspectos clave a desarrollar por el profesor:

- Diseño de los materiales que va a poner a disposición de los estudiantes.

- Preparación tanto de las tareas a realizar fuera del aula para obtener retroalimentación sobre lo que han o no aprendido los estudiantes, como para poner en práctica, en el aula, los conocimientos adquiridos.

Respecto a la elaboración de los materiales, sin descartar la utilización de fuentes de contenidos en textos escritos, se recomienda en el diseño del proceso de la clase inversa la utilización de materiales audiovisuales. Tienen la ventaja de que son mejor aceptados por los estudiantes (Spencer, 2011), ya que pueden ser visualizados en cualquier momento, con cualquier dispositivo móvil o tablet, tantas veces como quieran según sus necesidades y además se amplian las posibilidades de compresión y análisis con los mismos. Se aconseja que no tengan duración superior a 10 minutos, así como evitar la saturación que puede producir en el estudiante que tenga que visualizar un número excesivo de vídeos para cada sesión.

En cuanto a las tareas para llevar a cabo la supervisión del trabajo autónomo no presencial del estudiante es aconsejable, para garantizar el éxito, la preparación de una serie de preguntas o test sencillos que tras visualizar los vídeos puedan proporcionar retroalimentación al profesor a la vez que informar a los estudiantes sobre el grado de adquisición de los conocimintos concretos. Estas preguntas deben formularse con el fin de garantizar la adquisición de resultados de aprendizaje de niveles cognitivos más bajos según la taxonomía de Bloom, como son: recordar, comprender, aplicar, etc.

Las actividades que se diseñen para llevar a cabo, de manera presencial, en el aula deben estar orientadas a obtener resultados de aprendizaje correspondientes a los niveles cognitivos superiores de taxonomía de Bloom, como son: resolución de ejercicios de manera individual y en equipo que impliquen analizar, razonar, evaluar, etc.

En base a estas premisas se diseñó el proceso a seguir para llevar adelante la experiencia:

a) Elección de un tema del programa.

b) Creación de materiales audiovisuales para la experiencia.

(cc) EY-NC-ND 2016, Universitat Politècnica de València 
c) Elaboración de la guía de trabajo con detalles de las actividades a realizar fuera del aula y los plazos de entrega.

d) Uso de la herramienta Tareas de PoliformaT para registrar las entregas.

e) Corrección de tareas y envío de retroalimentación a los estudiantes.

f) Diseño de las actividades para realizar en el aula.

g) Preparación de un cuestionario para que los estudiantes reflexionen y valoren la experiencia.

\subsection{El desarrollo de la experiencia}

a) Para la elección del tema del programa se tuvo en cuenta tanto la duración del mismo como el momento en que se imparte en el curso. Se eligió un tema de duración corta, que se imparte en la cuarta semana de clase.

b) Para la creación de materiales audiovisuales se hizo uso de la herramienta "Videoapuntes" diseñada por la UPV, cuya imagen puede verse en la Figura 2. Se disponía de grabaciones de videoapuntes de todas las clases de los cursos 2013-14 y 2014-15, de manera que se eligieron las mejores grabaciones para exportar tantos vídeos como conceptos se incluyen en el tema de estudio. Se generaron 8 vídeos con una duración media de 9,5 minutos.

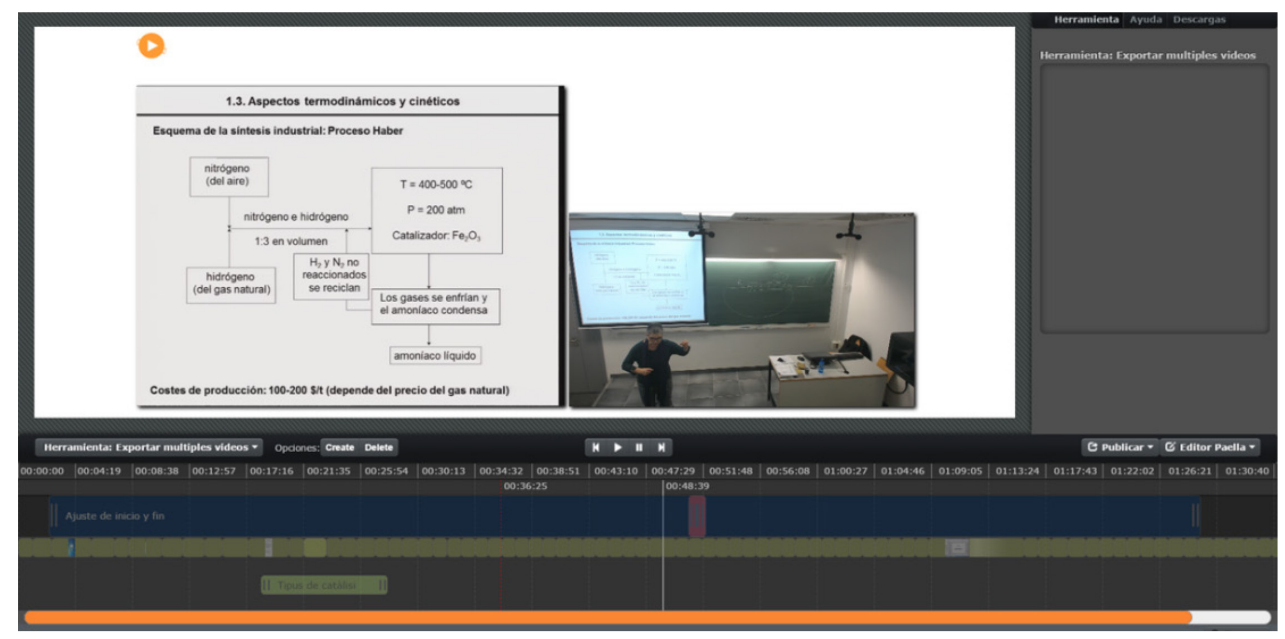

Fig. 2 Herramienta de Videoapuntes

c) Se elaboró la guía de trabajo con detalles de las actividades a realizar fuera del aula y los plazos de entrega. Se plantearon 3 tareas que consistían en la visualización de 2 o 3 vídeos, según la tarea, y la contestación de 4 cuestiones de respuesta múltiple por cada una de las tareas. El plazo de entrega de las tareas fue de 2 o 3 días, según la tarea, de manera que se pudo realizar la experiencia en una semana (incluido el fin de semana), tal como se tenía previsto. Se les pidió también a los estudiantes que indicasen el tiempo de realización de

(c)) 2016, Universitat Politècnica de València

Congreso In-Red (2016) 
cada una de las tareas con el fin de reprogramarlas, en caso de necesidad, para posteriores ocasiones.

d) Para entregar las tareas realizadas se hizo uso de la herramienta "Tareas" de la plataforma educativa de la UPV "PoliformaT". Así se pudo obtener el registro de la entrega, en plazo o no, de las respuestas a las cuestiones. La comprobación de la visualización de los vídeos se realizó mediante la opción disponible en la propia herramienta de "Videoapuntes".

e) La corrección de tareas y el envío de retroalimentación se realizó de manera muy rápida, antes de las 12 horas después de finalizar el plazo de entrega de cada tarea. Esto permitió la detección de errores conceptuales antes de la clase de aula con el fin de proceder a la aclaración de los mismos y la resolución de las dudas planteadas por los estudiantes.

f) Las actividades a realizar en el aula consistieron en la resolución en equipos de trabajo de ejercicios y cuestiones de nivel de complejidad elevado. Para cada ejercicio se procedió a solicitar a los equipos:

- participación en el planteamiento razonado de las propuestas de resolución

- planteamiento de las dudas

- resolución colectiva de las dudas

y finalmente la resolución detallada y el análisis de los resultados en el grupo completo de la clase.

g) Finalizada la experiencia se pasaron a los estudiantes dos cuestionarios, uno de escala Likert y otro de respuesta abierta. El cuestionario cuantitativo consistió en una serie de preguntas relacionadas con la planificación, los materiales, la elección adecuada de tareas, la utilidad para el aprendizaje, el ritmo de las clases, el grado de satisfacción, etc. Respecto al cuestionario cualitativo se plantearon preguntas para la reflexión y la valoración de la experiencia sobre los aspectos positivos y de mejora, la conveniencia de hacer extensivo al resto de la materia y/o a otras asignaturas, etc. (Ver apartado 3.3).

\section{Resultados}

\subsection{Realización de las tareas fuera del aula.}

El análisis de los datos obtenidos sobre la participación en la realización de las tareas indica que los 41 estudiantes matriculados en el grupo realizaron las tareas 1 y 2 en el plazo marcado. Respecto a la tarea 3, el $80 \%$ entregó la tarea en plazo, resultado que se considera muy satisfactorio habida cuenta de que tenían un control de una asignatura justo al día siguiente de la fecha que se les indicó para la entrega. Cabe estacar que el $40 \%$ de los estudiantes que no pudieron entregar en plazo esta última tarea la enviaron en las 24 horas siguientes.

Los resultados sobre el porcentaje de respuestas acertadas a las 4 preguntas que se planteaban en cada una de las tareas pueden verse en la Figura 3. Destaca el elevado porcentaje de entregas con 4 y 3 respuestas acertadas (entre el 85 y el $90 \%$ ) en todas las tareas.

(c) EY-NC-ND 2016, Universitat Politècnica de València 


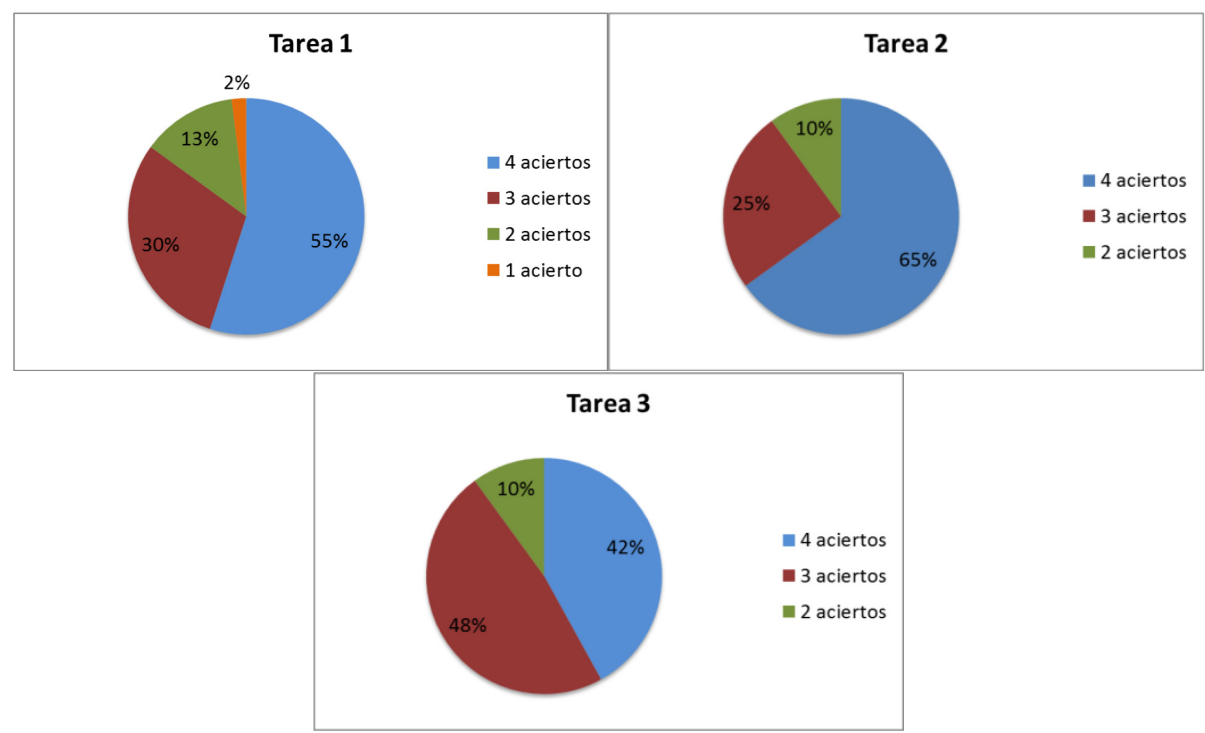

Fig. 3 Porcentaje de respuestas acertadas en las entregas de las tareas 1-3

\subsection{Asistencia y participación en las actividades de las sesiones presenciales en el aula.}

Se registró la asistencia a las sesiones presenciales en el aula y se pudo constatar que excepto 2 personas, que justificaron la ausencia, asistieron todos. La participación en la resolución de problemas, planteamiento de preguntas y verbalización de las respuestas fue muy activa y regulada. A destacar el respeto en los turnos de palabra.

\subsection{Valoración de los estudiantes de la experiencia de la clase inversa.}

Los cuestionarios cuantitativos y cualitativos para valorar la experiencia fueron contestados por los 41 estudiantes matriculados en el grupo. En el cuestionario cuantitativo se analizó el número de respuestas en cada uno de los cinco niveles de la escala Likert de cada ítem y en el cualitativo de respuesta abierta, todas las manifestaciones en cada una de las preguntas.

Los resultados obtenidos en el análisis de las respuestas al cuestionario de escala Likert sobre preguntas relacionadas con el proceso (instrucciones recibidas, planificación y secuenciación de las tareas, materiales empleados) y las cuestiones enunciadas para profundizar sobre el aprendizaje de los conceptos, así como el ritmo con que se han desarrollado las clases en el aula se muestran en la Figura 4. 


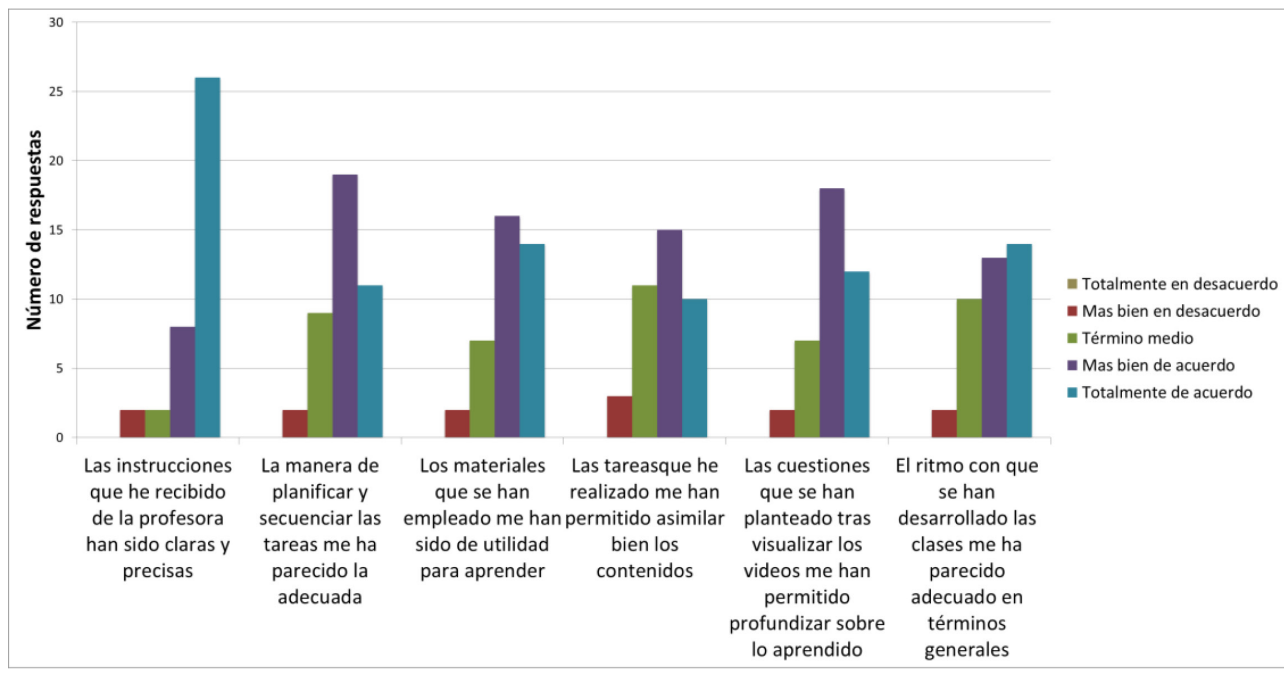

Fig. 4 Valoración de los estudiantes sobre el proceso

Resulta destacable que, en un aspecto tan importante para lograr el éxito como es que los estudiantes tengan muy claro qué es lo que han de hacer y cómo deben realizarlo, el $85 \%$ manifiesta estar totalmente de acuerdo o más bien de acuerdo en que la profesora les ha dado instrucciones claras y precisas. El $75 \%$ indica que está totalmente de acuerdo o más bién de acuerdo en la planificación realizada, los materiales empleados y las cuestiones planteadas en las tareas. Finalmente, alrededor del $65 \%$ opina que las tareas les han permitido asimilar los contenidos y que el ritmo de las clases ha sido adecuado. Este porcentaje, menor que los anteriores, se puede justificar en base a la heterogeneidad en el nivel de conocimientos previos que tienen los estudiantes lo que puede influir en la dificultad de algunos de ellos para construir conocimiento y para seguir el ritmo normal de las clases.

En términos generales se puede afirmar que el proceso seguido ha permitido, a un gran número de estudiantes, alcanzar el objetivo de adquirir los conocimientos necesarios mediante las tareas, que de manera autónoma y activa, han realizado fuera del aula, con los materiales que se les ha proporcionado. Asímismo, las clases que se han desarrollado para aplicar los conocimientos adquiridos se han seguido, con bastante satisfacción por un porcentaje elevado de estudiantes.

Por lo que respecta a la valoración de la experiencia, en el cuestionario cuantitativo sobre preguntas relacionadas con el nivel de aprendizaje adquirido, la satisfacción personal, el tiempo empleado y la percepción sobre el desarrollo de competencias, los resultados se muestran en la Figura 5.

Si observamos las respuestas referidas a totalmente de acuerdo o más bien de acuerdo se destaca que los estudiantes admiten, en un porcentaje bastante elevado (70\%), que el esfuerzo que han realizado para aprender ha valido la pena. En el resto de manifestaciones hay ciertas discrepancias, y se destaca la variación de respuestas que se obtienen acerca de,

(c) ) EY-NC-ND 2016, Universitat Politècnica de València 
si han aprendido más y mejor con la metodología inversa, y sobre si el tiempo invertido para aprender los conceptos ha sido mayor que si se hubiese desarrollado la clase magistral, aunque, como puede verse en la Figura 5, está sesgada hacia el extremo de totalmente de acuerdo. Esto confirma que cada estudiante tiene sus propias estrategias de aprendizaje y prefiere una metodología u otra y que el tiempo que cada persona requiere para aprender es diferente en función de su nivel de conocimientos previos.

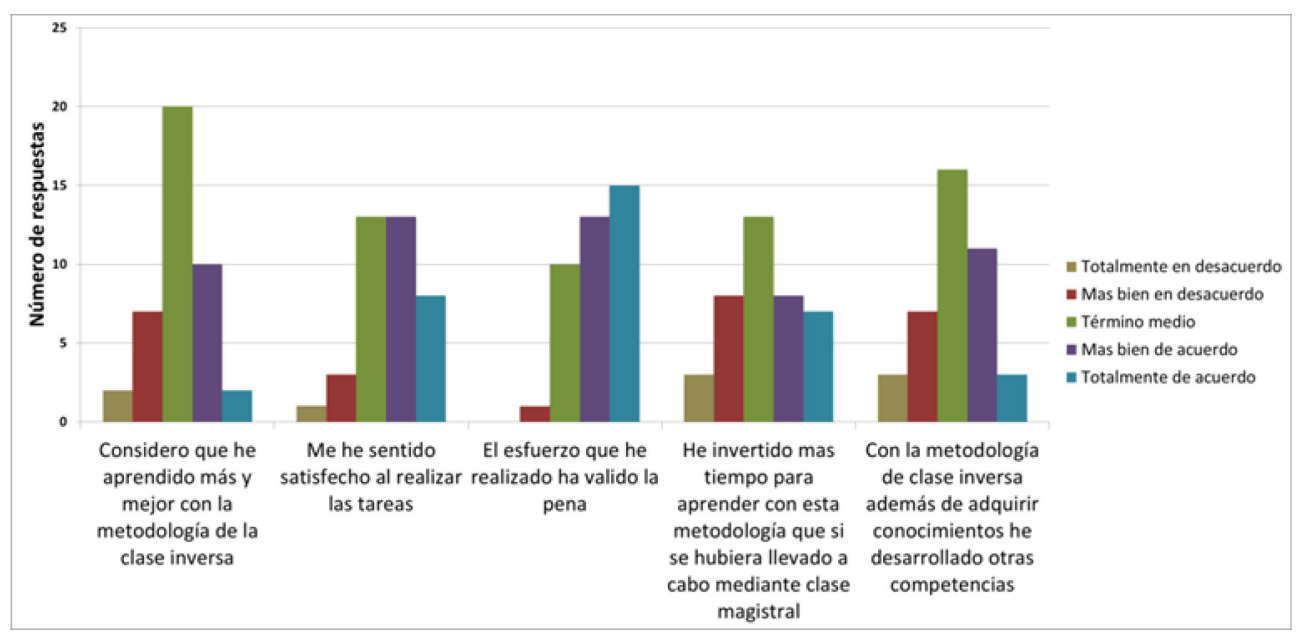

Fig. 5 Valoración de los estudiantes sobre su percepción respecto al aprendizaje y satisfacción personal

Por otra parte, también se observa la discrepancia de respuestas en lo referido a la percepción de los estudiantes sobre la posibilidad de desarrollar otras competencias además de adquirir conocimientos al implementar la clase inversa, aunque su percepción está sesgada hacia el extremo de acuerdo o conformidad.

Finalmente, se destaca que un elevado número de estudiantes manifiesta su satisfacción al realizar las tareas lo que, unido a sus respuestas sobre si el esfuerzo ha valido la pena, pone de manifiesto que cuando los estudiantes aprenden por ellos mismos se sienten especialmente satisfechos y ello les permite comprobar que, con esfuerzo personal pueden conseguir lo que se proponen.

Respecto al cuestionario cualitativo en el que se plantearon preguntas para la reflexión y la valoración de la experiencia sobre los aspectos positivos y de mejora (entre paréntesis se indica la frecuencia de la respuesta), el valor añadido que consideran que tienen esta metodología y la conveniencia de hacerla extensiva al resto de la materia y/o a otras asignaturas, los resultados se presentan en la Tabla 1. 
Hacia la Clase Inversa. Una Experiencia de Aprendizaje de la Química y de Desarrollo de Competencias en el Primer Curso de Grado en Ingenierías

Tabla 1. Opiniones de los estudiantes sobre la experiencia de la clase inversa

\begin{tabular}{|c|c|}
\hline ASPI & MEJORAS A IMPLEMENTAR \\
\hline 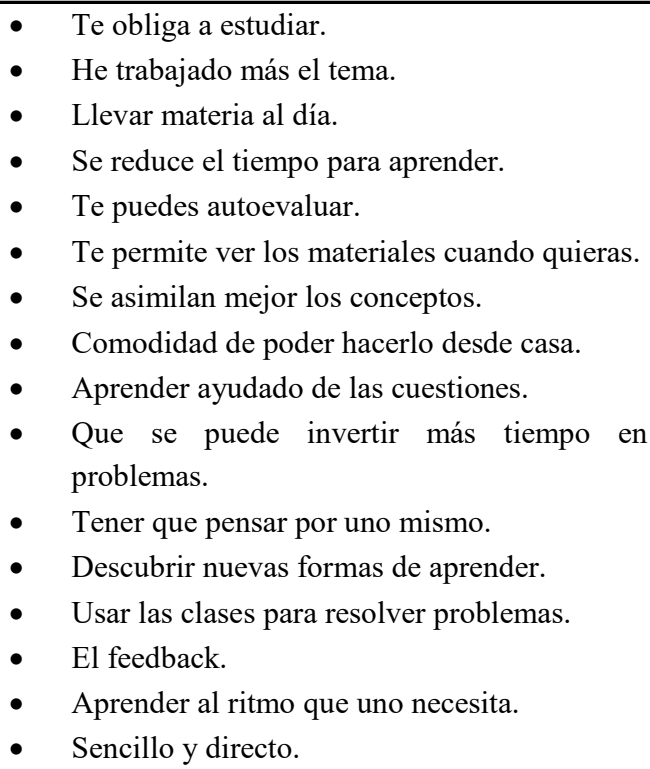 & $\begin{array}{l}\text { - } \quad \text { No cambiaría nada (10). } \\
\text { - } \quad \text { Más tiempo (4). } \\
\text { - } \text { Añadir más cuestiones a las tareas } \\
\text { (4). } \\
\text { - } \text { Más ejercicios (2). } \\
\text { - Hacerlo voluntario. }\end{array}$ \\
\hline VALOR AÑADIDO DE LA METODOLOGÍA & $\begin{array}{c}\text { CONVENIENCIA DE HACERLA } \\
\text { EXTENSIVA A TODA LA MATERIA }\end{array}$ \\
\hline $\begin{array}{l}\text { - } \quad \text { Te hace más responsable. } \\
\text { - } \quad \text { Más esfuerzo personal y poner de tu parte. } \\
\text { - } \quad \text { Te obliga a estudiar todos los días. } \\
\text { - } \quad \text { Se aprende más pero a cambio de tiempo. } \\
\text { - } \quad \text { Te hace autodidacta. } \\
\text { - } \\
\text { - Indenonomía. } \\
\text { - A tu ritmo. }\end{array}$ & $\begin{array}{l}\text { - No. } \\
\text { - Me resulta más fácil la clase } \\
\text { "corriente". } \\
\text { - Los conceptos los entiendes mejor si } \\
\text { la profesora los explica en clase. } \\
\text { - Me gustan más clases dinámicas de } \\
\text { la profesora. } \\
\text { Algunos temas sí por romper la } \\
\text { monotonía. }\end{array}$ \\
\hline
\end{tabular}

En la Tabla 1, destaca la variedad de aspectos positivos que enumeran los estudiantes y la coincidencia en bastantes de ellos en que no cambiarían nada de la experiencia, ya que la ven bien así. Algunos indican la necesidad de dedicarles más tiempo y añadir más cuestiones a las tareas y más ejercicios para aplicar los conceptos (aspectos a tener en cuenta para la implementación en el siguiente curso).

Respecto a la pregunta formulada para provocar la reflexión sobre el valor añadido que creen tiene esta metodología, destaca que los que responden lo hacen marcando las ideas de: "responsabilidad", "esfuerzo personal", "organización del tiempo", "aprendizaje autónomo", es decir, competencias transversales que sin duda se ejercitan y se desarrollan con este tipo de enfoque pedagógico.

En general, los resultados obtenidos están de acuerdo con las reflexiones de Tourón, Santiago y Díez quienes, en las diferentes referencias citadas, coinciden en destacar la

(cc) EY-NC-ND 2016, Universitat Politècnica de València 
importancia del seguimiento del contenido del curso, cada uno a su ritmo, para lograr una mejora de la comprensión conceptual, así como de la implicación y del compromiso de los estudiantes en el aprendizaje. Además, las opiniones de los estudiantes coinciden con las de Tourón y Santiago (2015) cuando indican que en el modelo de clase inversa, además de producirse un aprendizaje más consolidado y duradero, se promueve el desarrollo de competencias, proceso en el que el profesor, por su papel de orientador y facilitador del aprendizaje, es más necesario que nunca (Tourón, 2014).

Finalmente indicar que, es notorio que una parte importante de los que respondieron la pregunta sobre la conveniencia de hacer la clase inversa extensiva a toda la materia manifestaron que "no" aunque no todos argumentaron los motivos por los que decidieron dar esta respuesta; los que sí lo hicieron, coincidieron en que sería mucho trabajo fuera del aula el que deberían realizar. Al preguntarles sobre la conveniencia de utilizar la clase inversa en otras asignaturas, los que contestaron coincidieron en la asignatura de Física, probablemente por ser una materia en la que disponer del tiempo de clase para realizar ejercicios de aplicación, relación, justificación, etc., lo perciben como muy conveniente.

\section{Conclusiones}

Del análisis de los resultados y de las opiniones de los estudiantes sobre la experiencia se puede concluir que:

- Los estudiantes se han sentido auténticos protagonistas de su aprendizaje, ya que todos se han implicado en las tareas, y, salvo alguna excepción, han cumplido los plazos de entrega y han respondido correctamente a las cuestiones sobre los contenidos que de manera autónoma han aprendido.

- El diseño del proceso ha sido el apropiado, tanto en lo referido a la guía de trabajo, a los materiales creados o a las actividades a realizar fuera y dentro del aula. Algunos alumnos recomiendan añadir algunas cuestiones y ejercicios y disponer de algo más de tiempo para la realización de las tareas para el siguiente curso.

- Se ha conseguido provocar la reflexión sobre su propio aprendizaje ya que todos han contestado los cuestionarios que, al respecto, se habían elaborado.

- Los estudiantes aprecian la labor llevada a cabo por la profesora en el diseño y desarrollo de la experiencia de la clase inversa: manifiestan estar satisfechos y reconocen que les ha sido útil para aprender.

- Son muchos los aspectos positivos de la experiencia que los estudiantes destacan, mientras que son pocos los que consideran que se deben mejorar.

- Se han conseguido desarrollar diferentes competencias transversales, entre las que destaca el trabajo autónomo, la organización del tiempo y la capacidad para asumir la responsabilidad y el protagonismo en el aprendiazje, al tiempo que han aprendido Química.

- He obtenido con esta experiencia la retroalimentación necesaria para mejorar el diseño de la misma y aprovechar el potencial de esta técnica para hacerla extensiva a otras partes de la asignatura. 
Hacia la Clase Inversa. Una Experiencia de Aprendizaje de la Química y de Desarrollo de Competencias en el Primer Curso de Grado en Ingenierías

\section{Referencias}

ANDERSON, L. W. and KRATHWOHL, D. R. (Eds.) (2001). A Taxonomy for Learning, Teaching, and Assessing: A Revision of Bloom's Taxonomy of Educational Objectives. Allyn \& Bacon. Boston, MA.

BERGMANN, J., OVERMYER, J., and WILLIE, B. (2011). The flipped class: What it is and What it is not. The Daily Riff. Disponible en http://www.thedailyriff.com/articles/the-flipped-classconversation-689.php [Consulta: 29 de marzo de 2016].

BERGMANN, J. and SAMS, A. (2012). Flip your Classroom: Reach Every Student in Every Class Every day. Washington, DC: ISTE.

BLOOM, B. S. and KRATHWOHL, D. R. (1956). Taxonomy of Educational Objectives: The Classification of Educational Goals. Handbook I: Cognitive Domain. New York: Longmans Green.

SPENCER D., WOLF D. and SAMS A. (2011) Are you ready to flip? The Daily Riff. Disponible en http://www.thedailyriff.com/articles/are-you-ready-to-flip-691.php [Consulta: 1 de abril de 2016].

GARGALLO, B. et al. (2014). "Metodología centrada en el aprendizaje. Su impacto en las estrategias de aprendizaje y en el rendimiento académico de los estudiantes universitarios". Revista Española de Pedagogía, $\mathrm{n}^{\mathrm{o}} 259$, p. 415-435.

GARGALlO, B., MORERA, I. y GARCÍA-FÉLIX. E. (2015). "Metodología innovadora en la universidad. Sus efectos sobre los procesos de aprendizaje de los estudiantes. Anales de Psicología, 31(3), p. 901-915.

MAZUR, E. (1996) Peer Instruction: A User's Manual, New York: Prentice Hall.

TOURÓN, J. y SANTIAGO, R. (2013). “Atención a la diversidad y desarrollo del talento en el aula. El modelo DT-PI y las tecnologías en la implantación de la flexibilidad curricular y el aprendizaje al propio ritmo". Revista Española de Pedagogía, n 256, p. 441-459.

TOURÓN, J. y SANTIAGO, R. (2015). "El modelo Flipped Learning y el desarrollo del talento en la escuela”. Revista de Educación, no 368, p. 196-231.

TOURÓN, J., SANTIAGO, R. y DÍEZ, A. (2014). The flipped classroom. Cómo convertir la escuela en un espacio de aprendizaje. Barcelona: Digital-text.

(cc) EY-NC-ND 2016, Universitat Politècnica de València 